\title{
Study on the Usability of a Haptic Menu for 3D Interaction
}

\author{
Giandomenico Caruso, Elia Gatti, and Monica Bordegoni \\ Dipartimento di Meccanica, Politecnico di Milano, Via La Masa, 1 \\ 20156 Milano, Italy \\ \{giandomenico.caruso, elia.gatti, monica.bordegoni\} apolimi.it
}

\begin{abstract}
The choice of the interaction menu to use is an important aspect for the usability of an application. In these years, different solutions, related to menu shape, location and interaction modalities have been proposed. This paper investigates the influence of haptic features on the usability of 3D menu. We have developed a haptic menu for a specific workbench, which integrates stereoscopic visualization and haptic interaction. Several versions of this menu have been developed with the aim of performing testing sessions with users. The results of these tests have been discussed to highlight the impact that these features have on the user's learning capabilities.
\end{abstract}

Keywords: Mixed Reality, Haptic Interaction, Haptic Menu.

\section{Introduction}

The interaction menu is still an important factor in the usability of several kinds of applications. The type of menu to be used is strictly dependent on the purposes of the application, and on the input devices used for the interaction. Often 2D menus are also used in $3 \mathrm{D}$ applications, where the interaction is enabled by a $2 \mathrm{D}$ input device. However, when 3D input devices are used, 2D menus become less functional while the use of 3D menus simplifies the selection of menu items.

In recent years, different 3D-menu solutions have been developed by varying the shape, the location and the interaction modality. Among those, particularly interesting are the haptic menus. The use of haptic menus in three-dimensional applications seems to improve the user's capability in selecting the menu items since the physicalization of the menu increases its affordance. Several studies have investigated the benefits provided by this kind of menu in terms of item selection, task-execution time, accuracy, etc. In [1] the authors have compared different interaction metaphors for the selection of seven menu options using a haptic device with a pen interface. Their results have showed that the haptic feedback is useful for reducing the selection error rate and the choice of a specific interaction metaphor can sensibly improve the whole selection task. More detailed quantitative studies have explored the value of the haptic interaction with several parts of the desktop [2-4].

In the research presented in this paper we have investigated the features that can improve the usability of a 3D haptic menu, thus leading to the speeding up in the execution of some tasks. Different versions of the haptic menu have been developed

P. Campos et al. (Eds.): INTERACT 2011, Part II, LNCS 6947, pp. 186-193, 2011.

(C) IFIP International Federation for Information Processing 2011 
with the aim of performing several testing sessions with users. The tests have allowed us to check the impact that these features have on the user's learning capabilities. The paper describes the functioning of our haptic menu, the testing sessions, which have been conducted to validate it, and the elaboration and synthesis of the collected data.

\section{The 3D Haptic Menu}

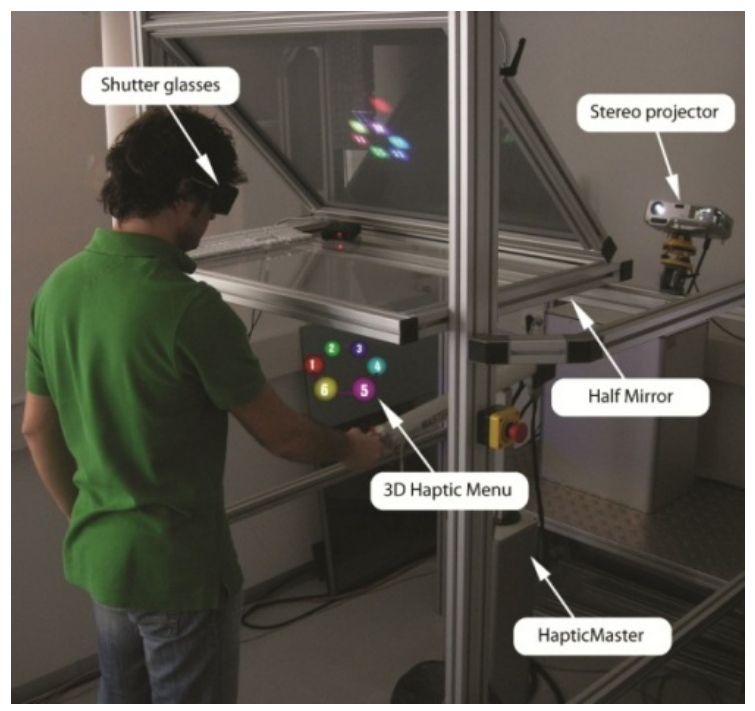

Fig. 1. A user during the interaction with the 3D Haptic Menu (montage)

The 3D Haptic Menu (3DHM), which has been investigated in our study, has been developed for being used with a specific workbench. The workbench consists of a stereoscopic visualization system, which is based on mirrors and screens for projecting a 3D image over the haptic workspace [5], and of the HapticMaster (HM) device made by MOOG [6]. The end effector of the HM is linked with a 3D cursor that allows interacting with the $3 \mathrm{D}$ objects. The purpose of this workbench is to enable the user with the haptic shape modification of large 3D models in real scale. Consequently, an interactive menu is necessary to activate the different modeling functions.

The shape of the 3DHM has been based on the pie menus that, with the right combination of selection method and assistive forces, can provide an excellent solution for 3D haptic environments [7]. The effectiveness of the pie menu approach has been also validated by a study carried out with blind users [8]. In the pie menu, the items are arranged in a circular design around the perimeter of the circle. The items of our 3DHM are represented by six colored spheres, which have the same diameter of the HM end effector $(44.5 \mathrm{~mm})$, and are connected by a purple torus. Fig. 1 shows the stereoscopic workbench and a user during the interaction with the 3DHM.

The haptic feedback of the 3DHM has been created basing on the primitive entities provided by the HM. A haptic torus limits the user's movement around the menu 
while each menu item is haptically rendered as a magnetic point (snap). The magnetic point is a haptic object made up of three haptic springs with the same application point but different stiffness and deadband. The first spring $S_{l}$ is an omnidirectional haptic spring with stiffness $K_{l}=250 \mathrm{~N} / \mathrm{m}$ without deadband. The second spring $S_{2}$ is another omnidirectional haptic spring with a higher opposite stiffness $K_{2}=-2500 \mathrm{~N} / \mathrm{m}$ and a deadband $D_{2}=0.0250 \mathrm{~m}$. With these two springs the attractive force remains constant outside the sphere, which represents the snap volume, while, if the HM end effector goes into the sphere, it remains locked, since $S_{2}$ is stiffer than $S_{1}$. The $S_{3}$ haptic spring, instead, has stiffness $K_{3}=-\left(K_{2}-K_{1}\right)=2250 \mathrm{~N} / \mathrm{m}$ and the deadband is $D_{3}=D_{2} \cdot K_{2} /\left(K_{2}-K_{1}\right) \approx 0.0277 m$. The role of $S_{3}$ is to reduce the action field of the attractive force to a thickness equal to $D_{3}-D_{2}$, and to cancel the sum of the other two haptic springs outside the snap volume. The final effect perceived by the user through the HM end effector is a magnetic attraction in the nearby of a menu item that facilitates the user to select it.

We have elaborated four different versions of the 3DHM to perform our usability study. The four versions have been named as follows: no-haptic, normal, contextual and contextual no-snap. The no-haptic version does not provide the user with haptic feedback and the menu item selection is only highlighted by means of a different illumination of the item. The normal version only returns the magnetic points as haptic feedback. In the contextual version torus and magnetic points are enabled and the position of the menu is defined according to the HM end effector position, i.e. the first menu item position is the same of the HM end effector. The high external stiffness of the torus compels the HM end effector to move only into the menu. The contextual no-snap version is similar to the contextual one but only the torus haptic feedback is rendered. Table 1 summarizes the four versions of the 3DHM.

Table 1. The four versions of the 3DHM.

\begin{tabular}{|l|c|c|c|}
\hline 3DHM version & torus & snap & position \\
\hline no-haptic & disabled & disabled & centered \\
\hline normal & disabled & enabled & centered \\
\hline contextual & enabled & enabled & relative \\
\hline contextual no-snap & enabled & disabled & relative \\
\hline
\end{tabular}

\section{Evaluation Tests}

When people interact with a simple menu, the task they perform could be interpreted as a point-to-point movement, similar to those described in [9], involving the sensorimotor skills. Visual information, such as the menu and the cursor position, and kinesthetic information, as the position of the hand, are integrated to create a motor program that permits users to move the cursor to a predefined point. In neuroscience the structure of this sensory integration and the building of this kind of motor program has been widely discussed [10-12]. In our study, we have assumed that the user first localizes the position of the menu item, which he has to select, and the position of the cursor. Then, he codifies such coordinates in the extrinsic space and subsequently these coordinates have to be translated into the intrinsic space, enabling the user's 
motor system to execute the task. When the transformation has been made, the user plans the trajectory needed to reach the menu item. All the steps of these coordinate transformations are well described in [9] and summarized in Fig.2.

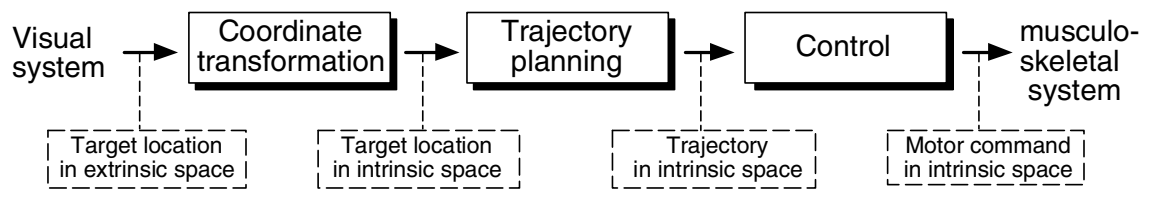

Fig. 2. Adaptive Internal Model of Intrinsic Kinematics [9]

The aim of the evaluation tests has been to assess whether the position and the haptic features of the 3DHM can facilitate the building and the memorability of the motor program needed to reach a menu target. To perform the evaluation tests, four different users' groups, one for each 3DHM version, have been involved to perform a simple task (between group experimental design). Each group consists of 5 users (20 users in total), all males, right handed and aged between 24 and 35. The users are naïve to the task, and had no previous experience with the haptic menu. The tests have been repeated in 6 different sessions, to evaluate whether and how the users' ability in interacting with a particular version of the menu changed over time. The task consisted in a simple menu item selection. When a user selected one of the menu items a white sphere appeared in the 3D space and specifically in a position related to the item selected. Then, the user had to reach that sphere and select it with the cursor. The sequence of menu items to select has been read from a list by the experimenter. After this selection, the white sphere disappeared and the experimenter read from the list the number corresponding to the next item that the user had to select. The session ended when the user selected all the 30 items listed in the experimenter's list. The number of items in the list was balanced per item (6 items per 5 repetitions) and randomized. The users have completed the whole sessions in a week.

\subsection{Results}

The task to accomplish has been really easy and intuitive: no one of the testers made selection errors during the testing sessions. The learnability of the menus has been assessed by analyzing the selection time during the six sessions. Since we were not interested in the time needed to complete the task, but only in the time in which the testers were able to select the right menu item, only the time occurring between the click of the white sphere and the successive menu item selection has been elaborated. Those intervals have been summed for each session, in order to have the total selection time that resulted equal to the time that the user spent in selecting the right menu item during the entire session. During the testing sessions also the user's trajectory path has been recorded. Fig. 3 shows a trajectory path elaborated from a very brief part of a testing session. In particular, the red sphere indicates the position where the user activates the menu, the colored spheres are the menu items selected and the white spheres are the target spheres. This path has been used to extract some 


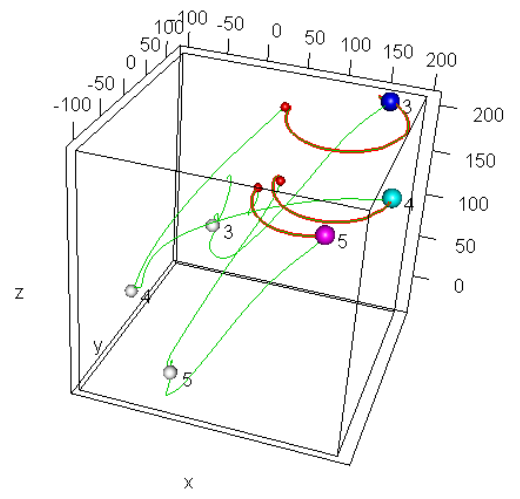

Fig. 3. A trajectory path elaborated from a testing session

general information about user's movements. We have elaborated such data to assess if the selection of a menu item can be assimilated to a generic reaching task, as we previously assumed. In particular, by analyzing the velocity profile, which has been obtained between two subsequent selections, we have observed bell-shaped curves.

In order to evaluate the usability and the memorability in using the different menus, we have evaluated how the total selection time varied per each tester and per each menu during the six sessions. The sphericity of the data set has been assessed through the Mauclhy test $(p$-value $>0.05)$ and then a two way repeated measures ANOVA has been applied to analyze the variation of the selection time along the sessions. The ANOVA's factors taken into account are the position of the menus (contextual vs. fixed) and the presence of the snap feedback (present, absent). The kind of torus has not been considered, because the conditions (absent vs. high) were collinear with the position factor. That has been a forced choice, since to the choice enabling a torus in a fixed menu where users directly reached the item did not make sense, whereas in a contextual menu the presence of the torus could be useful (see discussion). However, further studies will be aimed to disambiguate those conditions. The analysis showed a statistically significant interaction between the haptic feedback and the position of the menu between users $\left(F_{1,16}=5.4163\right.$, p-value $\left.<0.05\right)$. In particular, post-hoc t-test comparisons at $95 \%$ of confidence interval showed a highly statistical difference between the fixed no haptic menu and other versions ( $p$-value < 0.001 in all cases). A strong effect of repetition $\left(F_{5,80}=11.1619\right.$, p-value $\left.<0.001\right)$ and an effect of the interactions repetition: haptic feedback $\left(F_{5,80}=2.6106, p\right.$-value $<$ $0.05)$ and repetition: menu position $\left(F_{5,80}=3.3479\right.$, p-value $\left.<0.01\right)$ have been found within participants. Fig. 4a shows how the users' performance changes along the repetitions for each group of users. Lines have been drowned only to evidence a general trend in the data. Fig. 4b shows the standard error for each kind of menu in each session. As a general comment, the contextual haptic menu seems to have a more homogenous dataset, whereas the fixed haptic standard menu generally a grater variance of the results. 


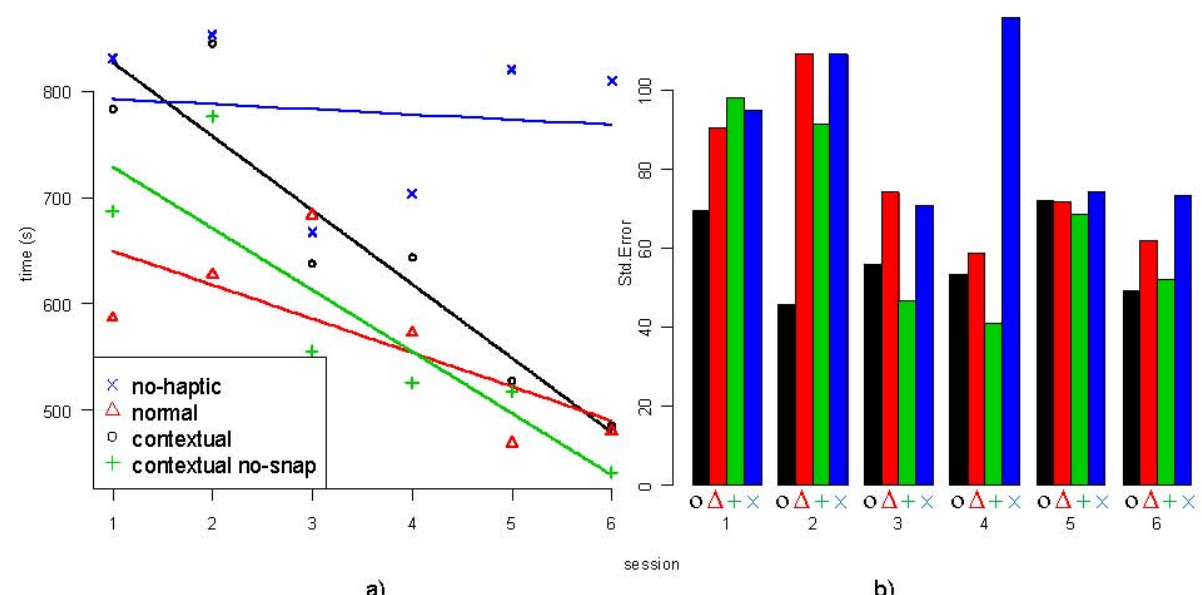

Fig. 4. a) Means of each group in each session. b) Standard error for each group and session

We also collected some impressions from the users who interacted with the different versions of the menus. The main feedback we received was about the haptic feedback modality, which was requested in the case of the non-haptic menu. Moreover, most of the users found disturbing the snap attraction in the contextual modality.

\section{Discussion}

In the above-described testing sessions, the user's learnability and usability of the different versions of our 3DHM have been evaluated. The test has been structured by assimilating the menu item selection to a reaching task. Our assumption has been supported by the analysis of the trajectories and the velocity profiles that are, in all cases, similar to those examined in [14] and assume characteristics as those of a reaching task.

The two-way repeated measures ANOVA showed statistical significant differences between subjects belonging to different groups. In particular, post-hoc comparisons showed that in the no-haptic menu the performance times are different (higher) from those obtained when using the other menu versions. This is due to the fact that the total absence of a haptic feedback made difficult for users to reach the menu items. In other words, they found difficult to reach the items basing only on the visual feedback. In a 3D scene, indeed, it is important to have as much information as possible about the position of the object. The haptic modality provides a feedback in this direction, whereas the visual modality can be (at least in absence of external landmark, as in our case) insufficient. Furthermore, within effects showed that the learning ability (interaction between repetition and the other factors) marginally depends on the kind of snap haptic feedback (present/not present) but considerably on the position in which the menu appeared.

Our initial hypothesis was that a haptic menu, which simplifies the motor program involved in a reaching task, could improve the usability and the learnability of the 
menu itself. To understand how and to what extent the position and the haptic features influence the motor program simplification we have developed four versions of our 3DHM. The activation of a menu linked to the position in a 3D space of the user's hand facilitates the construction of a motor program. Linking the position of the menu to the position of the cursor, indeed, makes the users able to learn the position of the menu items in the intrinsic space, since it is assumed that the position of the cursor should be already in the intrinsic coordinates. Thus, learning the position of the menu items in the intrinsic coordinates facilitates the learning of the menu: actually, in that way the user can avoid performing the coordinate transformation step during the creation of the motor program. Moreover, the presence of the torus could be determinant in minimizing the errors in the menu item selection during learning, constraining the user's movement in a right plane: further studies will be aimed to support this hypothesis. The snap haptic feedback also appears to be slightly significant in the learning process. This is because extra kinesthetic cues, as the force feedback, could facilitate the tuning of the user attention on the proprioceptive cues, which are codified in intrinsic coordinates. Nevertheless, the best performance for the mean of execution time in different sessions has been obtained by the contextual nosnap version. This is probably due to the fact that in the contextual version, the presence of the snap points, although prevents a faster execution of the task, is useful for remembering the item position.

\section{Conclusion}

In this paper a new kind of haptic menu has been presented: the 3DHM. Starting from the studies concerning the building of motor programs [9-12] the usability and the learnability of 4 versions of the 3DHM have been tested, in order to assess whether the position and the haptic features of the 3DHM can facilitate the building and the memorability of the motor program needed to reach a menu target.

The data collected have highlighted the effectiveness of our investigation approach, based on the cognitive features of the user. In general, the haptic feedback improves the usability of a 3D menu both in terms of usability and learnability. Besides, we have assessed that there are significant differences related to the menu position in the 3D space: a contextual position makes the menu little intuitive in the first sessions but the performance, in term of task-execution times, sensibly increases.

We also assisted to an effect of the haptic feedback both in terms of usability and learnability. We conclude that in both the cases haptic feedback is a useful characteristic that should be implemented in 3D menus.

Due to the high variance of the collected data among the users, further studies are certainly necessary to achieve more significant results. In particular, we consider interesting to conduct supplementary tests, in blind conditions, to assess the influence of different haptic feedbacks in the memorability of a haptic menu and to clarify the role of the torus in the contextual version.

Acknowledgments. The authors would like to thank all the participants to the testing sessions and in particular Daniela De Lucia and Eleonora Bartoli for their precious contribution. 


\section{References}

1. Raymaekers, C., Coninx, K.: Menu Interactions in a Desktop Haptic Environment. In: Proc. Eurohaptics, Birmingham, UK, pp. 49-53 (2001)

2. Oakley, I., Adams, A., Brewster, S., Gray, P.: Guidelines for the Design of Haptic Widgets. In: Proc. 16th British HCI Group Annual Conference, London (2002)

3. Oakley, I., Brewster, S., Gray, P.: Solving Multi-Target Haptic Problems in Menu Interaction. In: Proc. CHI 2001 Conference on Human Factors in Computing Systems, Seattle, Washington, pp. 357-358 (2001)

4. Oakley, I., McGee, M.R., Brewster, S., Gray, P.: Putting the Feel in 'Look and Feel'. In: Proc. CHI 2000 Conference on Human Factors and Computing Systems, The Hague, The Netherlands, pp. 415-422 (2000)

5. Bordegoni, M., Covarrubias, M.: Direct Visuo-Haptic Display System Using a Novel Concept. In: Proc. 3th Eurographics Symposium on Virtual Environments (2007)

6. HapticMaster:

http: / /www.moog.com/products/haptics-robotics (accessed on June 2011)

7. Komerska, R., Ware, C.: A study of haptic linear and pie menus in a 3D fish tank VR environment. In: Proc. 12th International Symposium on Haptic Interfaces for Virtual Environment and Teleoperator Systems, pp. 224-231 (2004)

8. Sjöström, C.: Designing Haptic Computer Interfaces for Blind People. In: Proc. Sixth IEEE International Symposium on Signal Processing and its Applications, Kuala Lumpur, Malaysia (2001)

9. Imamizu, H., Uno, Y., Kawato, M.: Adaptive Internal Model of Intrinsic Kinematics Involved in Learning an Aiming Task. Journal of Experimental Psychology: Human Perception and Performance 24(3), 812-829 (1998)

10. Desmurget, M., Grafton, S.: Forward modeling allows feedback control for fast reaching movements. Trends in Cognitive Sciences 4(11), 423-431 (2000)

11. Plamondon, R., Alimi, A.M.: Speed/accuracy trade-offs in target-directed movements. Behavioral and Brain Sciences 20(2), 279-349 (1997)

12. Pelisson, D., Prablanc, C., Goodale, M.A., Jeannerod, M.: Visual control of reaching movements without vision of the limb. II. Evidence of fast unconscious processes correcting the trajectory of the hand to the final position of a double-step stimulus. Experimental Brain Research 62(2), 303-311 (1986)

13. Gazzaniga, M.S.: The new cognitive neuroscience. MIT Press, Cambridge (2000)

14. Digby, E., Gordon, B., Matthew, H.: The control of goal-directed limb movements: Correcting errors in the trajectory. Human Movement Science 18(2-3), 121-136 (1999) 